\title{
Compliance and Fatigue Life Analysis of $U$-shape Flexure Hinge
}

\author{
Jingjing LIANG*, Ruiqin LI*, Shaoping BAI**, Qing LI*, Fengping NING*, Shuhua KANG* \\ *School of Mechanical Engineering, North University of China, Taiyuan, China,03005, \\ E-mail: liruiqin@nuc.edu.cn (Corresponding author) \\ **Department of Mechanical and Manufacturing Engineering, Aalborg University, 9220 Aalborg, Denmark, \\ E-mail:shb@mp.aau.dk \\ crossref http://dx.doi.org/10.5755/j01.mech.25.6.22686
}

\section{Introduction}

The flexure hinge is a kind of special kinematic pair which makes use of the deformation of materials to provide finite angular displacement of complex movement around central axis. Compared with the traditional rigid structure, the flexure hinge has no friction, no abrasion, no clearance, low noise, small space size. The flexure hinge can be designed and fabricated in one body, which can realize high precision motion. The compliance can be used to protect the mechanism against impact amongst many other advantages. Flexure hinges are extensively applied in various fields requiring ultra-precision positioning, micromanipulation, microelectronics, and micro assembly for optoelectronic components, optics, bioengineering, and many other fields [1-3].

Up to date, researches on flexure hinge are mainly focused on the notch flexure hinge, reed flexure hinge, as well as flexible elements, such as rods, reeds, and the combination of flexure hinges with different notch shapes. The notch flexure hinge has higher precision but a smaller range of movement, which is more suitable for micro-displacement application. The reed flexure hinge has a larger rotation angle, but lower rotation accuracy. The combined hinge has better comprehensive performance [4-11].

Extensive researches on flexure hinges have been reported. Tian et al. proposed a V-type hinge structure, for which the compliance and rotation precision characteristics were analyzed by finite element method [12]. Chen et al. obtained two generalized models that are the conic model and the elliptic-arc-fillet model by using the ratio of the radius of curvature of the stress- concentrating feature to the minimum thickness as the only fitting variable [13]. Xu et. al. presented analytic models of four types of flexure hinges: elliptic, circular, parabolic, and hyperbolic. These analytic models are developed based on the theory of elasticity and infinitesimal method, and devoted a hinge index by the ratio of rotational precision and rotational stiffness, to estimate the mechanical properties of diverse flexure hinges synthetically and quantitatively [14]. Li et. al. developed a generalized analytical compliance model that can quickly formulate the equations of compliance and precision for hybrid flexure hinges [15]. Wang et. al. presented the development of a parametric model for the rotational compliance of a cracked right circular flexure hinge. The rotational compliance of the cracked right circular flexure hinge was obtained from the sum of the rotational compliance of a healthy flexure hinge and the change of the rotational compliance due to the crack [16]. Li et. al. presented a new type power-function-shaped flexure hinge, derived the closed-form compliance equations of the flexure hinge based on the unit-load method, and investigated the motion accuracy [17]. Meng et. al. investigated the existing stiffness equations for corner-filleted flexure hinges. Three empirical stiffness equations for cornerfilleted flexure hinges were formulated based on finite element analysis [18]. Li et. al. derived empirical compliance equations for circular flexure hinge considering the effect of stress concentration [19].

The notch flexure hinge is mostly applied to microoperation robots, magnifying mechanism for micrometric displacement, and compliance straight-line guidance mechanism, which has simple structure and can be processed as a whole. A thorough review of flexure hinges has indicated that different notch shape flexure hinges have been utilized to improve the performance. This paper presents a U-shape flexure hinge with four structures and the compliance equations for the flexure hinges. The influences of the structure parameters on the performance of these types of flexure hinges are investigated. It is found that the U-shape flexure hinges have a large compliance ranges corresponding to different notch size. This makes such flexure hinges capable of being used in wide potential applications with different requirements. The finite element analysis is used to comparing fatigue life of U-shape flexure hinges and circular flexure hinge, which reveals a longer fatigue life with U-shape design.

\section{Compliance analysis}

The U-shape flexure hinges are shown in Fig. 1, where four structural forms are displayed. In the flexures, notches are constructed with elliptic arc or circular arc connected by straight line segments.

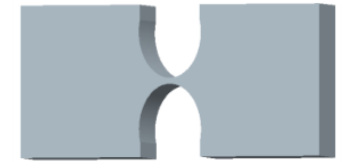

a

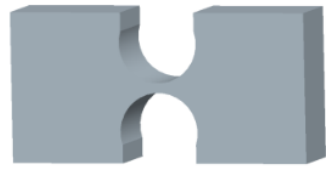

c

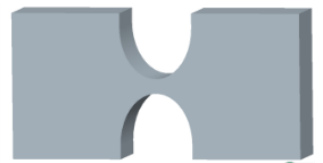

b

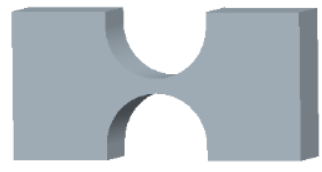

d
Fig. 1 Four kinds of U-shape flexure hinges with different structures: $\mathrm{a}-a \neq b$ and $2 b>m ; \mathrm{b}-a \neq b$ and $2 b=m$; $\mathrm{c}-a=b$ and $2 b>m ; \mathrm{d}-a=b$ and $2 b=m$ 
The structure parameters of the flexure hinge are shown in Fig. 2, including:

- the opening width $m$;

- the opening depth $n$ of straight-line segments;

- the semi major axis $a$ and semi minor axis $b$ of the ellipse;

- the minimum thickness $t$ of the flexure hinge center;

- the length $L$, width $W$ and height $H$ of the flexure hinge.

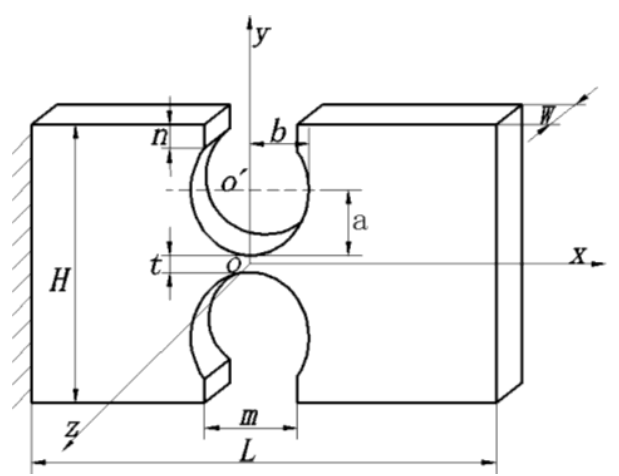

Fig. 2 Structural parameters of a U-shape flexure hinge

The compliance of the flexure hinges is mainly determined by the material and structural parameters. Thus, it is necessary to establish the relations between the compliance and structural parameters of the flexure hinge and reveal the influence of the structural parameters on the compliance to provide the theoretical basis for such engineering design and optimization of the flexure hinge. In the engineering application, the flexure hinge can have micro deformations in the displacement and the angle under the external load. In the analysis of compliance, it is assumed that the left end of the flexure hinge (being fixed) and the right end (being free) are each under the influence of the axial force $F_{x}$; shear forces $F_{y}, F_{z}$; and bending moments $M_{y}, M_{z}$ as shown in Fig. 3. The coordinate system $O-x y z$ is established with the geometric center of the hinge as the coordinate origin $O$.

The flexure hinge is subject to the above loads. According to Castigliano's second theorem [20]:

$$
\delta_{i}=\frac{\partial V_{\varepsilon}}{\partial F_{i}}
$$

where: $\delta_{i}$ is displacement corresponding to force $F_{i}, V_{\varepsilon}$, is structural deformation energy, $F_{i}$ is generalized force.

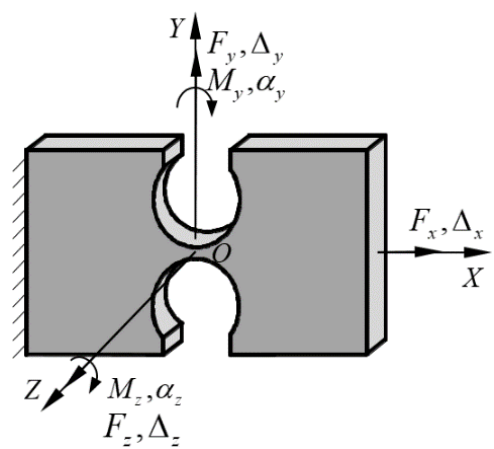

Fig. 3 The loads applied to U-shape flexure hinge

If $F_{i}$ is axial tension pressure, Eq. (1) becomes:

$$
\delta_{i}=\frac{\partial V_{\varepsilon}}{\partial F_{i}}=\frac{\partial}{\partial F_{i}} \int \frac{F_{N}{ }^{2}(x)}{2 E A} d x,
$$

where: $F_{N}$ is axial force, $E$ is modulus of elasticity, and $A$ is cross sectional area.

Otherwise, if $F_{i}$ is the bending moment, Eq. (1) is:

$$
\delta_{i}=\frac{\partial V_{\varepsilon}}{\partial F_{i}}=\frac{\partial}{\partial F_{i}} \int \frac{M^{2}(x)}{2 E I} d x,
$$

where: $M$ is bending moment, and $I$ is area moment of inertia.

A flexure hinge will generate five kinds of displacements, namely, linear displacements $x, y, z$ and angular displacements $\alpha_{y}, \alpha_{z}$ respectively, which can be obtained based on the theory of material mechanics about load-bearing and deformation relations as follows:

$$
\left[\begin{array}{c}
x \\
y \\
z \\
\alpha_{y} \\
\alpha_{z}
\end{array}\right]=\left[\begin{array}{ccccc}
C_{x-F_{x}} & 0 & 0 & 0 & 0 \\
0 & C_{y-F_{y}} & 0 & 0 & C_{y-M_{z}} \\
0 & 0 & C_{z-F_{z}} & C_{z-M_{y}} & 0 \\
0 & 0 & C_{\alpha_{y}-F_{z}} & C_{\alpha_{y}-M_{y}} & 0 \\
0 & C_{\alpha_{z}-F_{y}} & 0 & 0 & C_{\alpha_{z}-M_{z}}
\end{array}\right]\left[\begin{array}{c}
F_{x} \\
F_{y} \\
F_{z} \\
M_{y} \\
M_{z}
\end{array}\right] .
$$

For the convenience of analysis and calculation, region segmentation has been made for the notch part of the flexure hinge, as shown in Fig. 4.

In Fig. 4 , lengths $l_{A}, l_{B}$ and $l_{C}$ are calculated by:

$$
\begin{aligned}
& l_{A}(x)=2 a-2 a \cos \phi+t, \\
& d x=b \cos \phi d \phi, \phi \in\left[-\frac{\pi}{2}, \frac{\pi}{2}\right], \\
& l_{B}(x)=2\left[n+a \cos \phi_{0}-a \cos \phi\right],
\end{aligned}
$$

$$
d x=b \cos \phi d \phi, \phi \in\left[-\frac{\pi}{2},-\phi_{0}\right],
$$$$
l_{C}(x)=2\left[n+a \cos \phi_{0}-a \cos \phi\right],
$$$$
d x=b \cos \phi d \phi, \phi \in\left[\phi_{0}, \frac{\pi}{2}\right] .
$$$$
\text { Let } \frac{a}{t}=p, \quad s(\phi)=2 p-2 p \cos \phi+1, \frac{a}{n}=q,
$$ 
$k(\phi)=1+q \cos \phi_{0}-q \cos \phi$,

then: $l_{A}(x)=t \cdot s(\phi), \quad l_{B}(x)=l_{C}(x)=2 n \cdot k(\phi)$.

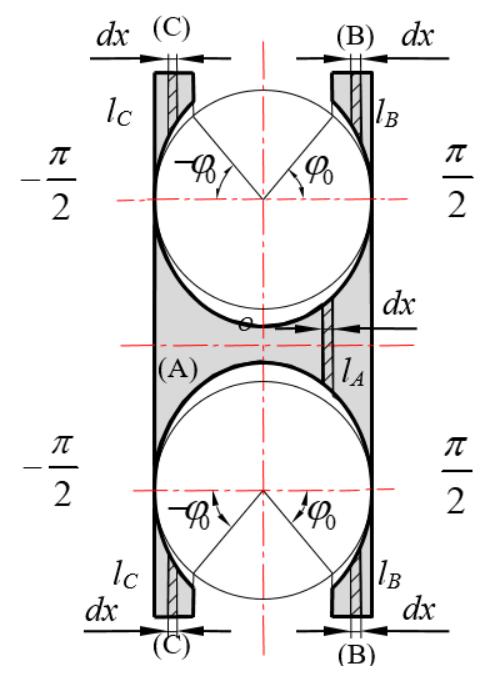

Fig. 4 Partition of the flexure hinge for compliance calculation

low:

The compliance equations can be derived as fol-

1) Angular compliance about $y$-axis and $z$-axis:

$C_{\alpha_{z}-M_{Z}}=\frac{12 b}{E W t^{2}} \int_{-\frac{\pi}{2}}^{\frac{\pi}{2}} \frac{\cos \phi}{[2 a(1-\cos \phi)+t]^{3}} d \phi+$

$+\frac{3 b}{E W n^{2}} \int_{\phi_{0}}^{\frac{\pi}{2}} \frac{\cos \phi}{\left[n+a \cos \phi_{0}-a \cos \phi\right]^{3}} d \phi$,

$C_{\alpha_{y}-F_{z}}=\frac{12 b^{2}}{E W t^{3}} \int_{-\frac{\pi}{2}}^{\frac{\pi}{2}} \frac{\cos \phi}{2 a(1-\cos \phi)+t} d \phi+$

$+\frac{12 b^{2}}{E W t^{3}} \int_{\phi_{0}}^{\frac{\pi}{2}} \frac{\cos \phi}{\left[n+a \cos \phi_{0}-a \cos \phi\right]^{3}} d \phi$,

$C_{\alpha_{z}-F_{y}}=\frac{12 b^{2}}{E W t^{2}} \int_{-\frac{\pi}{2}}^{\frac{\pi}{2}} \frac{\cos \phi}{[2 a(1-\cos \phi)+t]^{3}} d \phi+$

$+\frac{3 b^{2}}{E W n^{2}} \int_{\phi_{0}}^{\frac{\pi}{2}} \frac{\cos \phi}{\left[n+a \cos \phi_{0}-a \cos \phi\right]^{3}} d \phi$,

$C_{\alpha_{y}-M_{y}}=\frac{12 b}{E W t^{3}} \int_{-\frac{\pi}{2}}^{\frac{\pi}{2}} \frac{\cos \phi}{2 a(1-\cos \phi)+t} d \phi+$

$+\frac{12 b}{E W t^{3}} \int_{\phi_{0}}^{\frac{\pi}{2}} \frac{\cos \phi}{n+a \cos \phi_{0}-a \cos \phi} d \phi$,

2) Linear compliance along $x$-, $y$ - and $z$-axis:

$C_{x-F_{x}}=\frac{b}{E W} \int_{-\frac{\pi}{2}}^{\frac{\pi}{2}} \frac{\cos \phi}{2 a(1-\cos \phi)+t} d \phi+$

$+\frac{b}{E W} \int_{\phi_{0}}^{\frac{\pi}{2}} \frac{\cos \phi}{n+a \cos \phi_{0}-a \cos \phi} d \phi$,

$$
\begin{aligned}
& C_{z-F_{z}}=\frac{24 b^{3}}{E W^{3}} \int_{-\frac{\pi}{2}}^{\frac{\pi}{2}} \frac{\cos \phi}{2 a(1-\cos \phi)+t} d \phi- \\
& -\frac{12 b^{3}}{E W^{3}} \int_{-\frac{\pi}{2}}^{\frac{\pi}{2}} \frac{\cos { }^{3} \phi}{2 a(1-\cos \phi)+t} d \phi+ \\
& +\frac{24 b^{3}}{E W^{3}} \int_{\phi_{0}}^{\frac{\pi}{2}} \frac{\cos \phi}{n+a \cos \phi_{0}-a \cos \phi} d \phi- \\
& -\frac{b^{3}}{E W^{3}} \int_{\phi_{0}}^{\frac{\pi}{2}} \frac{\cos { }^{3} \phi}{\left.n+a \cos \phi_{0}-a \cos \phi\right]^{3}} d \phi, \\
& C_{z-F_{z}}=\frac{24 b^{3}}{E W^{3}} \int_{-\frac{\pi}{2}}^{\frac{\pi}{2}} \frac{\cos \phi}{2 a(1-\cos \phi)+t} d \phi- \\
& -\frac{12 b^{3}}{E W^{3}} \int_{-\frac{\pi}{2}}^{\frac{\pi}{2}} \frac{\cos { }^{3} \phi}{2 a(1-\cos \phi)+t} d \phi+ \\
& +\frac{24 b^{3}}{E W^{3}} \int_{\phi_{0}}^{\frac{\pi}{2}} \frac{\cos \phi}{n+a \cos \phi_{0}-a \cos \phi} d \phi- \\
& C_{\alpha_{y}-F_{z}}=C_{z-M_{y}}, \quad \frac{\cos b^{3} \phi}{E W^{3}} \int_{\phi_{0}}^{\frac{\pi}{2}} \frac{C_{y-M z},}{n+a \cos \phi_{0}-a \cos \phi} d \phi, \\
& C_{n},
\end{aligned}
$$

Among Eqs. (1-9):

$$
\begin{gathered}
\phi_{0}=\arctan \frac{2 m b}{2 a \sqrt{4 b^{2}-m^{2}}} m \leq 2 b, \\
n=\frac{H}{2}-\left(a+\frac{t}{2}\right)-\frac{a}{2 b} \sqrt{4 b^{2}-m^{2}} .
\end{gathered}
$$

From Eqs. $(5 \mathrm{a}-\mathrm{i})$, it can be seen that the compliance of U-shape flexure hinge is inversely proportional to the elasticity modulus and the width of the hinge. Moreover, compliance is influenced by the parameters of the notch size. Upon setting the basic structural parameters $H$ and $W$ for the U-shape flexure hinge, its compliance can be expressed as a function of the notch sizes:

$$
C=C f(a, b, m, t)
$$

In the following section, the influence of the parameters of notch size on the compliance of the flexure hinges will be analyzed.

\section{Influences of the parameters of notch size on the com- pliance}

Without loss of generality, we assume that two of the parameters of notch sizes are variables and the other two parameters are constant. The structural parameters of the flexure joint are given as $H=30 \mathrm{~mm}, W=10 \mathrm{~mm}$.

When $m$ and $t$ are set as constant, $a$ and $b$ are variables, varying in the ranges of $a \in[4,5]$ and $b \in[2,5]$. When $a$ and $b$ are fixed, $m$ and $t$ are variables, varying in the ranges 
of $m \in[4,8], t \in[4,5]$. Then the relationship between compliance of the U-shape flexure hinge and the parameters of notch sizes $a, b$ and $m, t$ are plotted using Matlab, as shown in Fig. 5, a-g.

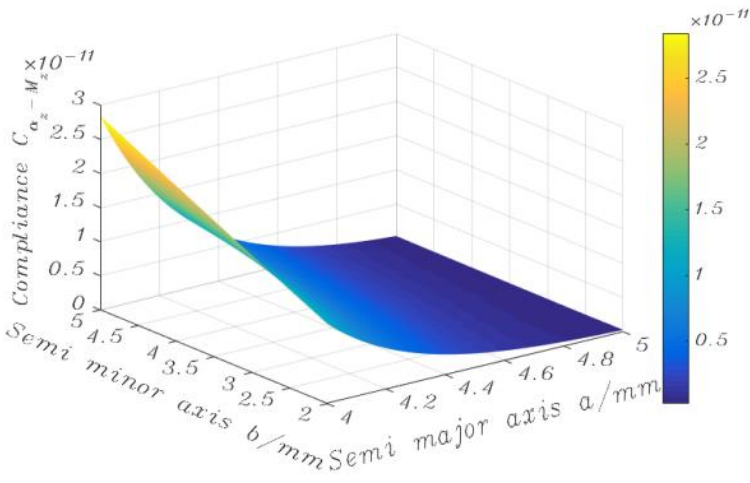

a

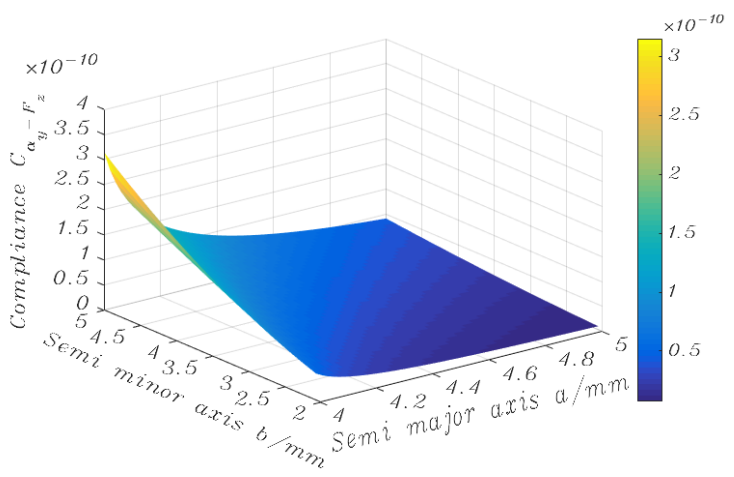

c

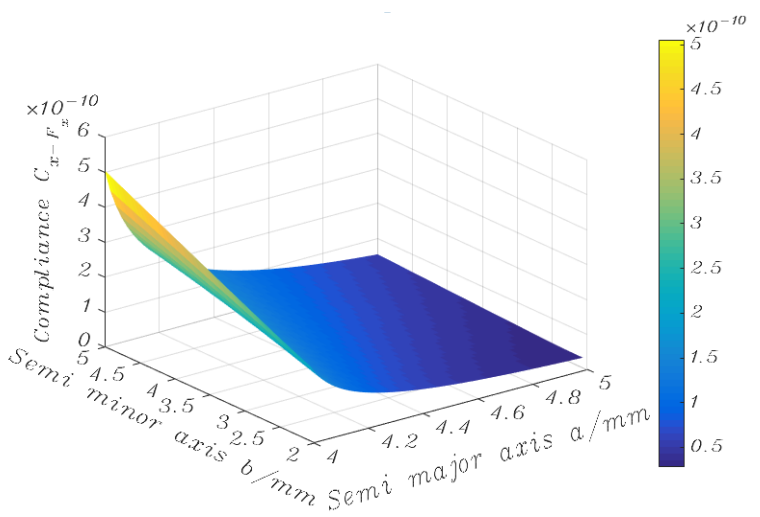

e
Fig. 5, a $-\mathrm{g}$ show each compliance of the U-shape flexure hinges with varying $a$ and $b$. We can observe from the plots characteristics, as described presently.

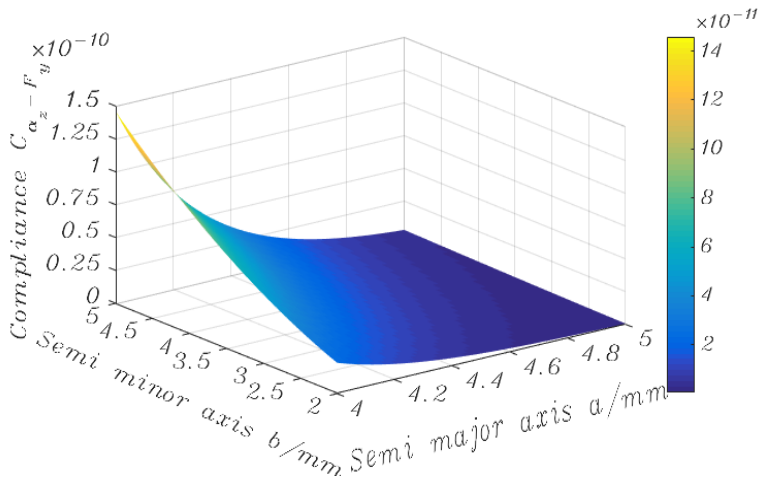

$\mathrm{b}$

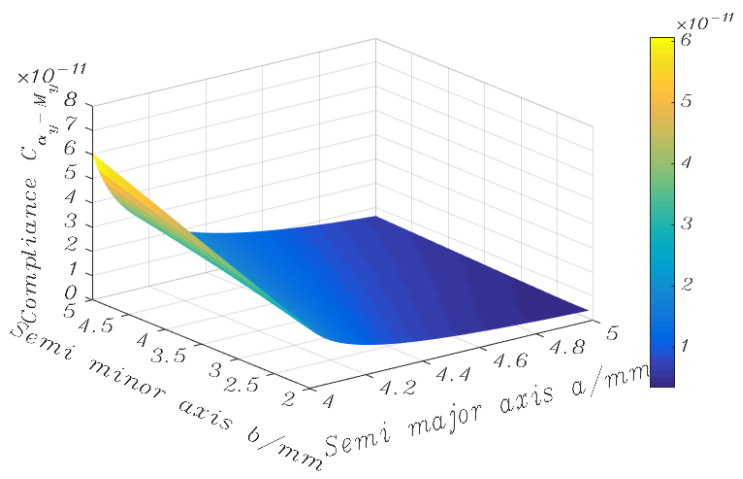

d

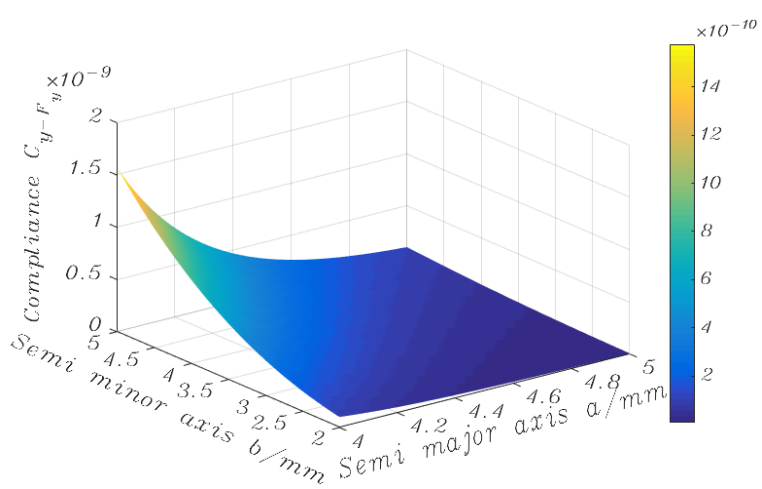

$\mathrm{f}$

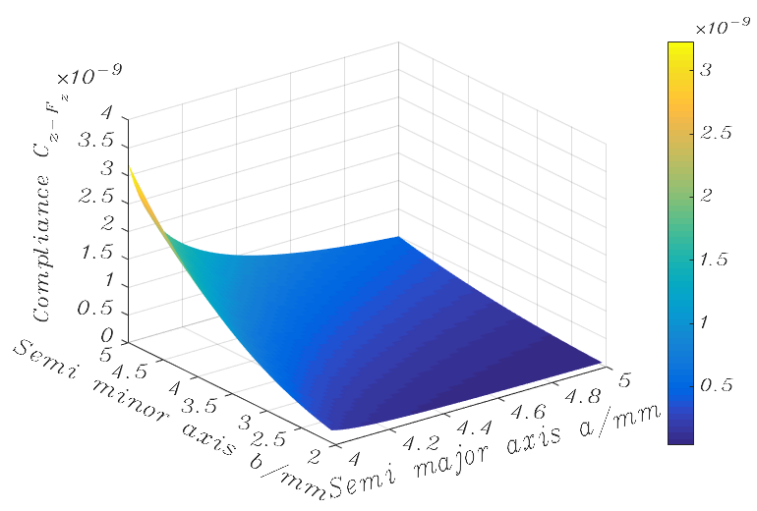

g

Fig. 5 The influence of parameters $a, b$ on compliance: a - compliance $C_{\alpha_{z}-M_{z}} ; \mathrm{b}-$ compliance $C_{\alpha_{z}-F_{y}} ; \mathrm{c}-$ compliance $C_{\alpha_{y}-F_{z}} ; \mathrm{d}$-compliance $C_{\alpha_{y}-M_{y}} ; \mathrm{e}-$ compliance $C_{x-F_{x}} ; \mathrm{f}-$ compliance $C_{y-F_{y}} ; \mathrm{g}-$ compliance $C_{z-F_{z}}$ 
When $a$ increases, the compliance decreases with $a$ nonlinearity. When $b$ increases, the compliance increases approximate linearly. The influence of $b$ incompliance is more significant. In each compliance, $C_{z-F_{z}}$ has the maxim-

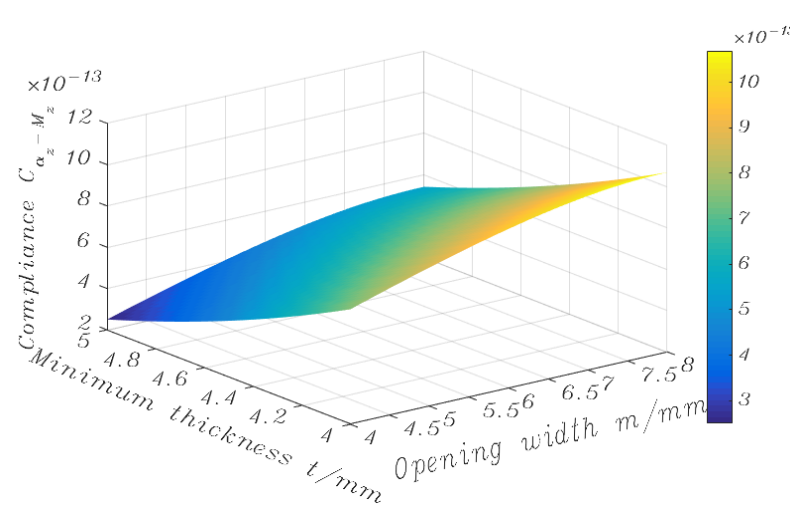

a

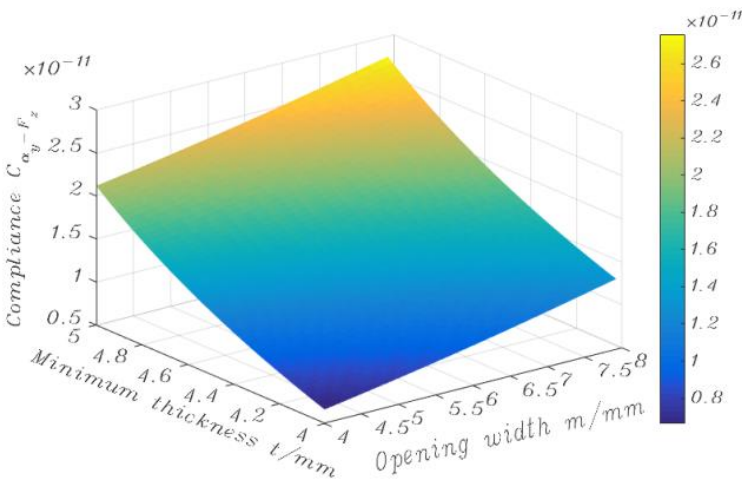

c

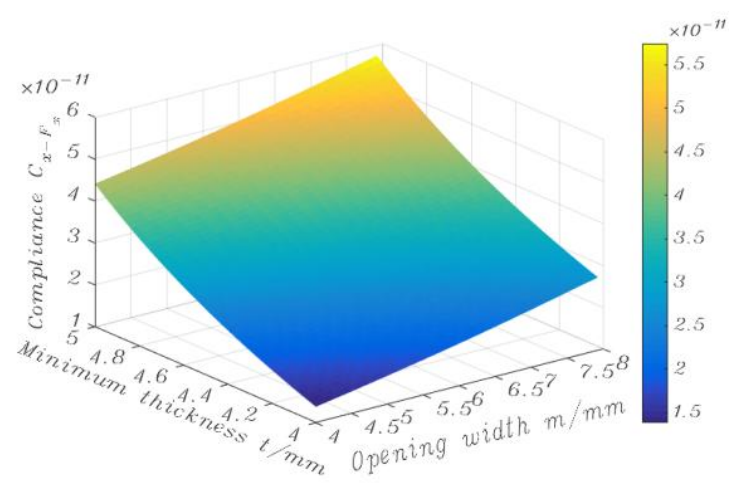

e um value and $C_{\alpha_{z}-M_{z}}$ has the minimum value within the value range of $a$ and $b$. When $a=5$ and $b=2$, each compliance reaches the smallest value.

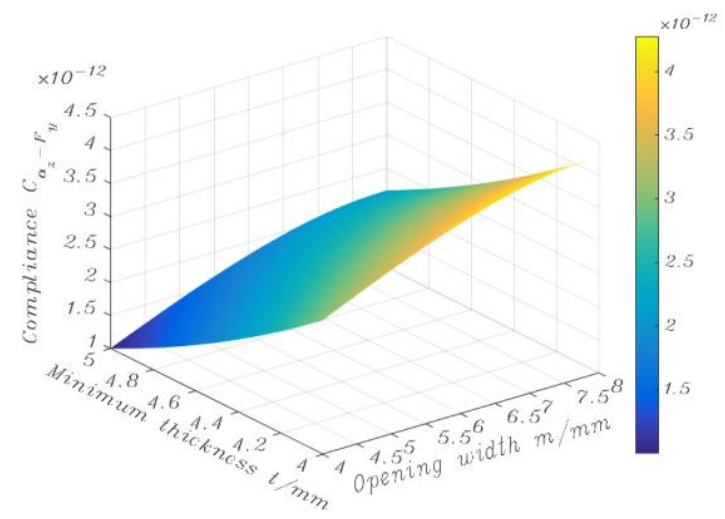

b

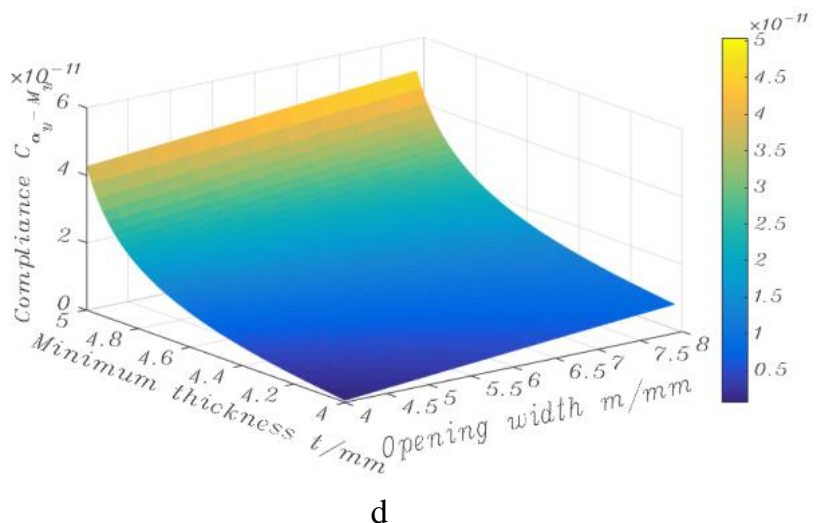

d

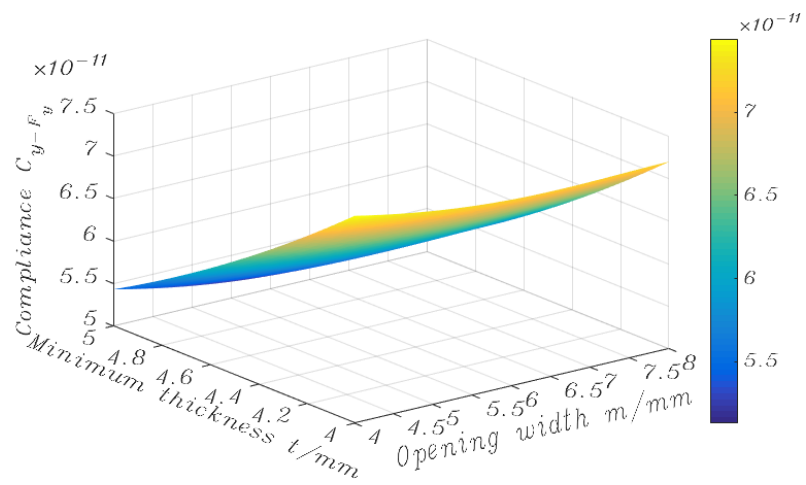

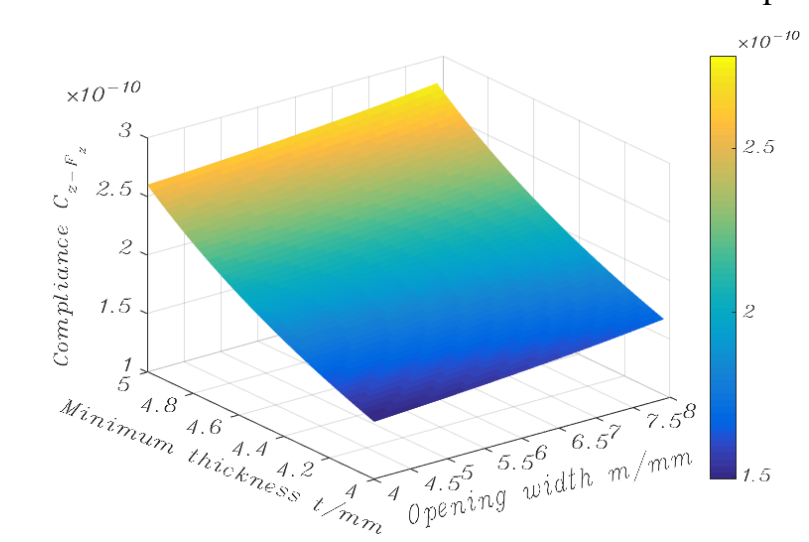

$\mathrm{g}$

Fig. 6 The influence of parameters $m, t$ on compliance when $a \neq b: \mathrm{a}-$ compliance $C_{\alpha_{z}-M_{z}} ; \mathrm{b}-$ compliance $C_{\alpha_{z}-F_{y}}$; c-compliance $C_{\alpha_{y}-F_{z}} ; \mathrm{d}$-compliance $C_{\alpha_{y}-M_{y}} ; \mathrm{e}$-compliance $C_{x-F_{x}} ; \mathrm{f}$-compliance $C_{y-F_{y}} ; \mathrm{g}$ - compliance $C_{z-F_{z}}$ 


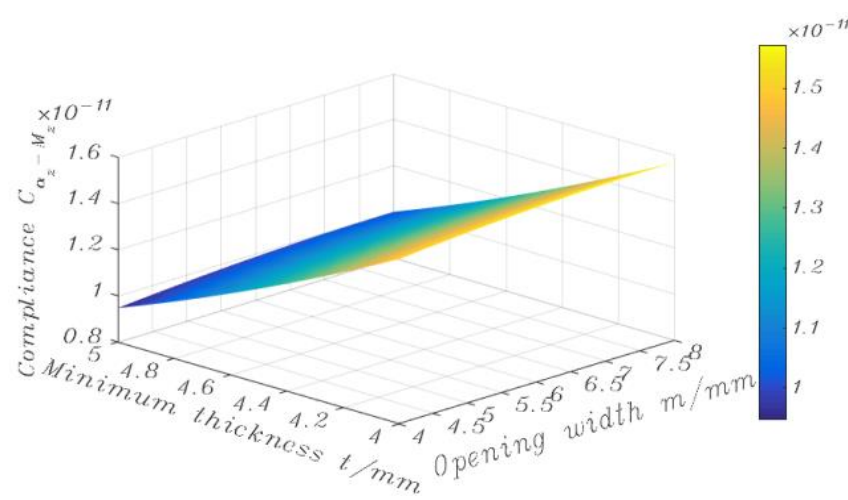

a

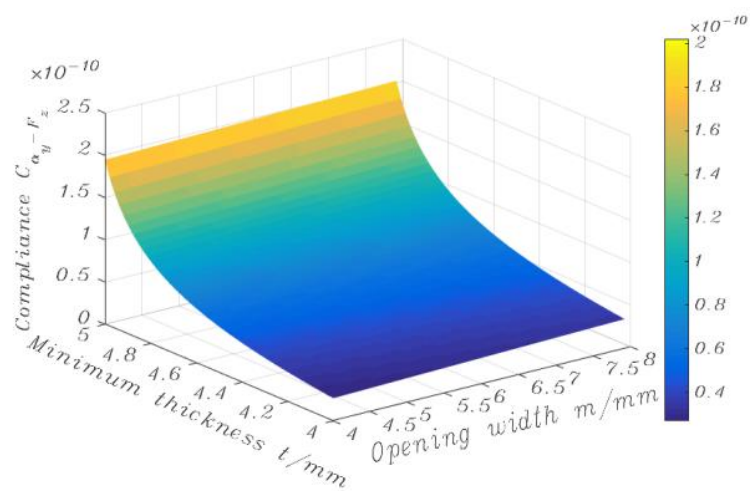

c

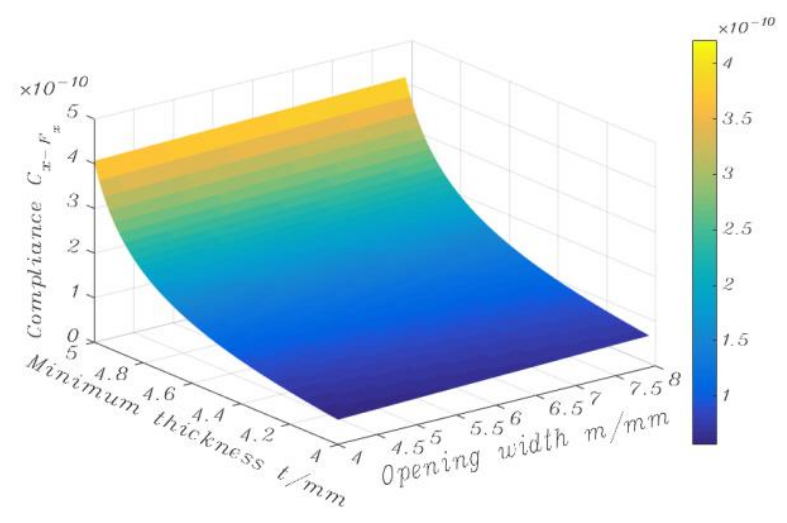

e

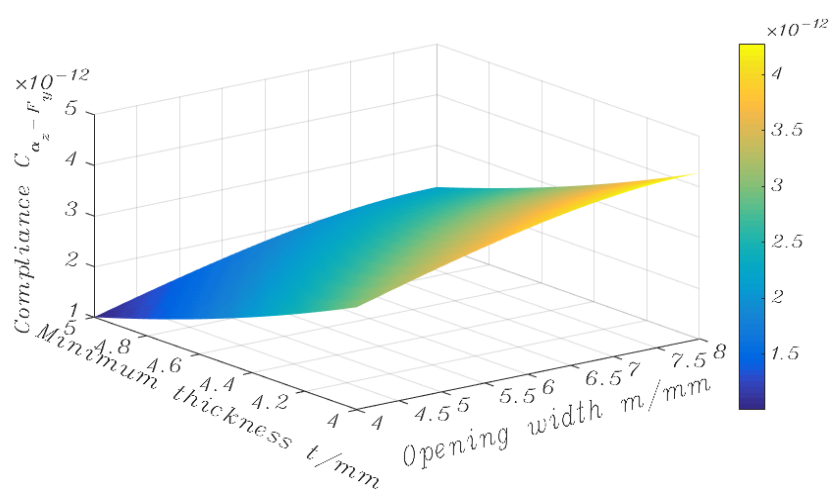

b

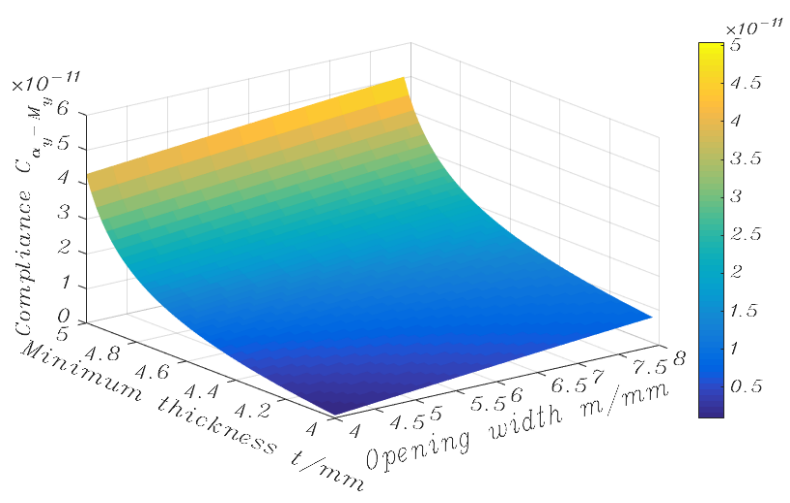

d

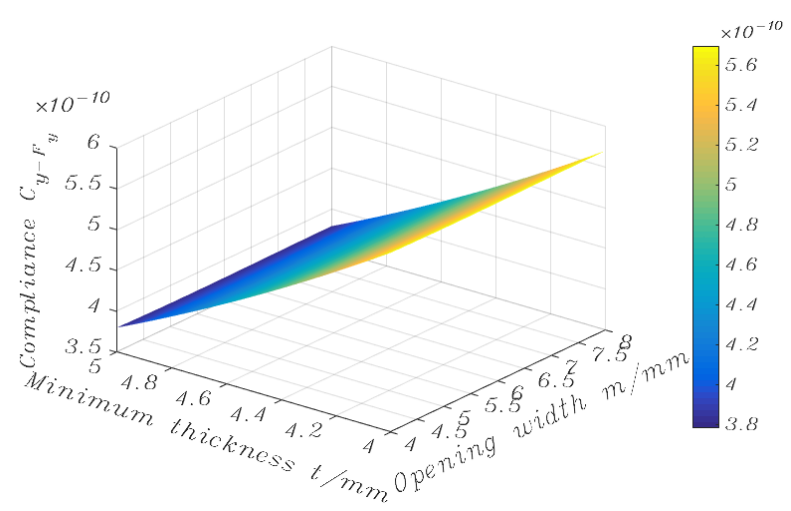

$\mathrm{f}$

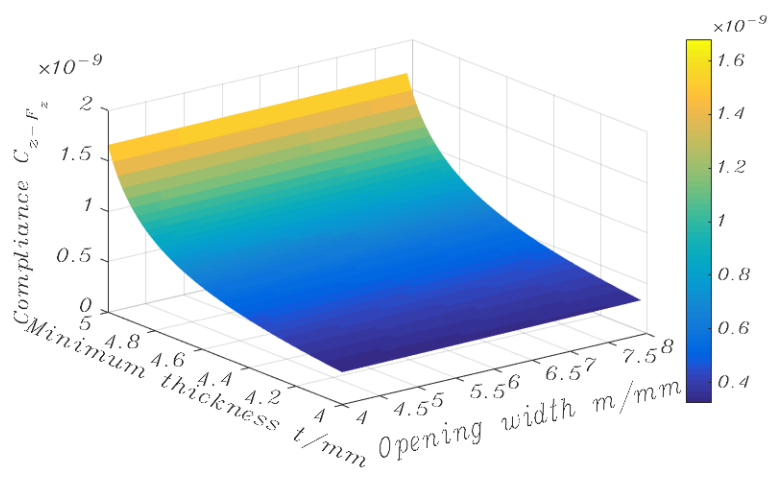

$\mathrm{g}$

Fig. 7 The influence of parameters $m, t$ on compliance when $a=b: \mathrm{a}-$ compliance $C_{\alpha_{z}-M_{z}} ; \mathrm{b}-$ compliance $C_{\alpha_{z}-F_{v}}$; $\mathrm{c}$-compliance $C_{\alpha_{y}-F_{z}} ; \mathrm{d}$-compliance $C_{\alpha_{y}-M_{y}} ; \mathrm{e}$-compliance $C_{x-F_{x}} ; \mathrm{f}$-compliance $C_{y-F_{y}} ; \mathrm{g}$-compliance $C_{z-F_{z}}$

Fig. 6 shows the compliance of the U-shape flexure hinges varying with respect to $m, t$ when $a \neq b$. Fig. 6 shows that the changes of the compliance of $C_{\alpha_{2}-M_{2}}$ (Fig. 6, a), $C_{\alpha_{z}-F_{y}}$ (Fig. 6, b) and $C_{y-F_{y}}$ (Fig. 6, f) are consistent with increase of $m$ and $t$, the compliance increases approximately 
linearly as $m$ increases, and the compliance decreases approximately linearly as $t$ increases. The change of the compliance of $C_{\alpha_{y}-F_{z}}$ (Fig. 6, c), $C_{x-F_{x}}$ (Fig. 6, e), and $C_{z-F_{z}}$ (Fig. 6, g) are consistent with the increase of $m$ and $t$. The compliance increases approximately linearly as $m$ and $t$ increase. The compliance $C_{\alpha_{y}-M_{y}}$ (Fig. 6, d) increases nonlinearly as $m$ and $t$ increase. When $m$ and $t$ are the smallest value in the range, the compliance $C_{\alpha_{y}-F_{z}}, C_{\alpha_{y}-M_{y}}, C_{x-F_{x}}$, and $C_{z-F_{z}}$ have the smallest value in the range. Fig. 7, a - g show the compliance of the U-shape hinge varying with respect to $m$ and $t$ when $a=b$. Fig. 7 shows that the changes of the compliance of $C_{\alpha_{z}-M_{z}}$ (Fig. 7, a), $C_{\alpha_{z}-F_{y}}$ (Fig. 7, b) and $C_{y-F_{\mathrm{y}}}$ (Fig. 7,f) are consistent with increase of $m$ and $t$, the compliance increases approximately linearly as $m$ increases, and the compliance decreases approximately linearly as $t$ increases. The change of the compliance of $C_{\alpha_{y}-F_{z}}$

(Fig. 6, c), $C_{\alpha_{y}-M_{y}}$ (Fig. 6, d), $C_{x-F_{x}}$ (Fig. 6, e), and $C_{z-F_{z}}$

(Fig. 6, g) are consistent with increase of $m$ and $t$, the compliance increases nonlinearly as $m$ and $t$ increase. When $m$ and $t$ are the smallest in the range, the compliance $C_{\alpha_{y}-F_{z}}$, $C_{\alpha_{y}-M_{y}}, C_{x-F_{x}}$, and $C_{z-F_{z}}$ have the smallest values in the range too.

\section{Simulation of fatigue life}

When the flexure hinge is applied to the flexible mechanism, the deformation of the mechanism is mainly caused by the large stress of the flexure hinge. The flexible mechanism usually uses piezoelectric ceramics to drive the flexure hinge to produce deformation. The mechanism failure is mainly reflected in the fatigue failure of flexure hinge. Therefore, it is necessary to study the fatigue life of flexure hinge to ensure the reliability of flexible mechanism.

The fatigue failure of flexure hinge under alternating load is generally occurred during the operation. Alternating loads produce alternating stress. Generally, alternating loads are decomposed into a load of constant amplitude and a load of variable amplitude. In this work, a periodic load of constant amplitude $10 \mathrm{~N}$ in the $\mathrm{X}$ and $\mathrm{Y}$ directions is considered. Material is structural steel with Young's Modulus 2e+011 Pa, Poisson's Ratio 0.3, Bulk Modulus $1.6667 \mathrm{e}+011 \mathrm{~Pa}$, Shear Modulus 7.6923e+010 Pa. The stress and cycle times are shown in Table 1. The fatigue life of U-shape flexure hinge was analysed by ANSYS simulation, and a series of fatigue life of U-shape flexure hinge with different notch size parameters were obtained, as listed in Table 2.

The main notch parameters of the U-shape flexure hinge are $a, b, m, t$. There are thirty models in three groups. The notch parameters are as follows:

$a=8, b=8, m=14$, while $t$ is variable;

$a=6, b=5, t=6$, while $m$ is variable;

$t=1, m=3$, while $a, b$ are variables.

The finite element model of the U-shape flexure hinge is built in the workbench. The left end is fixed, the right end loading (axial force, lateral force, bending moment), insert the fatigue analysis module Fatigue Tool and set the correction coefficient of fatigue analysis. The fatigue life cloud figure of the U-shape flexure hinge is shown in Fig. 8. Fatigue life is obtained, as shown in Table 2.

Curve fitting is carried out in Matlab. The influence curve of the notch parameter on the fatigue life of flexure hinge is obtained, as shown in Fig. 9, a - c.

Table 1

Stresses and cycles

\begin{tabular}{|c|c|}
\hline Alternating stress, $\mathrm{Pa}$ & Cycles \\
\hline $3.999 \mathrm{e}+009$ & 10 \\
\hline $2.827 \mathrm{e}+009$ & 20 \\
\hline $1.896 \mathrm{e}+009$ & 50 \\
\hline $1.413 \mathrm{e}+009$ & 100 \\
\hline $1.069 \mathrm{e}+009$ & 200 \\
\hline $4.41 \mathrm{e}+008$ & 2000 \\
\hline $2.62 \mathrm{e}+008$ & 10000 \\
\hline $2.14 \mathrm{e}+008$ & 20000 \\
\hline $1.38 \mathrm{e}+008$ & $1 . \mathrm{e}+005$ \\
\hline $1.14 \mathrm{e}+008$ & $2 . \mathrm{e}+005$ \\
\hline $8.62 \mathrm{e}+007$ & $1 . \mathrm{e}+006$ \\
\hline
\end{tabular}

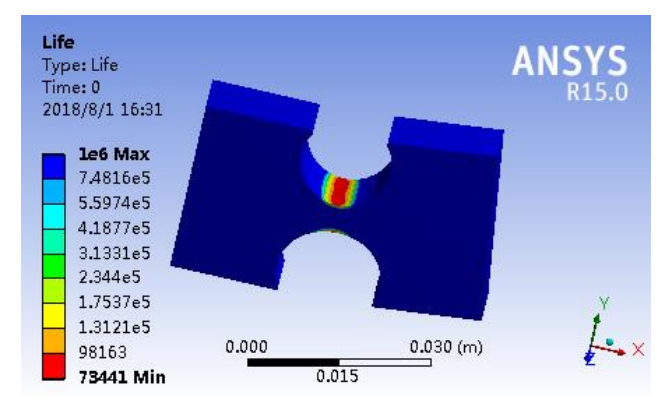

Fig. 8 Fatigue life cloud figure of U-shape flexure hinge with parameters $a=8, b=8, m=14, t=5$

Table 2

Fatigue life of U-shape flexure hinge under different incision parameters

\begin{tabular}{|c|c|c|c|c|c|}
\hline$a=8, b=8, m=14$ & $t=1$ & $t=2$ & $t=3$ & $t=4$ & $t=5$ \\
\hline$N(\log N)$ & $13.803(1.14)$ & $599.73(2.778)$ & $2706.6(3.4324)$ & $13090(4.1169)$ & $73441(4.8659)$ \\
\hline \hline$a=8, b=8, m=14$ & $t=6$ & $t=7$ & $t=8$ & $t=9$ & $t=10$ \\
\hline $\mathrm{N}(\log N)$ & $186060(5.2697)$ & $15545(4.1916)$ & $36157(4.5582)$ & $64214(4.8076)$ & $85065(4.9298)$ \\
\hline \hline$a=6, b=5, t=6$ & $m=3.5$ & $m=4$ & $m=4.5$ & $m=5$ & $m=5.5$ \\
\hline$N(\log N)$ & $146010(5.1644)$ & $145230(5.1621)$ & $146750(5.1666)$ & $145460(5.1627)$ & $147840(5.1698)$ \\
\hline \hline$a=6, b=5, t=6$ & $m=6$ & $m=6.5$ & $m=7$ & $m=7.5$ & $m=8$ \\
\hline$N(\log N)$ & $145190(5.1619)$ & $145470(5.1628)$ & $147800(5.1697)$ & $147230(5.168)$ & $147300(5.1682)$ \\
\hline \hline$t=1, m=3$ & $a=2.5, b=1.5$ & $a=3, b=2$ & $a=3.5, b=2.5$ & $a=4, b=3$ & $a=4.5, b=3.5$ \\
\hline$N(\log N)$ & $17.16(1.2345)$ & $16.537(1.2185)$ & $13.775(1.1391)$ & $13.861(1.1418)$ & $15.006(1.1763)$ \\
\hline \hline$t=1, m=3$ & $a=5, b=4$ & $a=5.5, b=4.5$ & $a=6, b=5$ & $a=6.5, b=5.5$ & $a=7, b=6$ \\
\hline$N(\log N)$ & $14.77(1.1694)$ & $14.633(1.1653)$ & $14.042(1.1474)$ & $13.975(1.1454)$ & $13.811(1.1402)$ \\
\hline
\end{tabular}




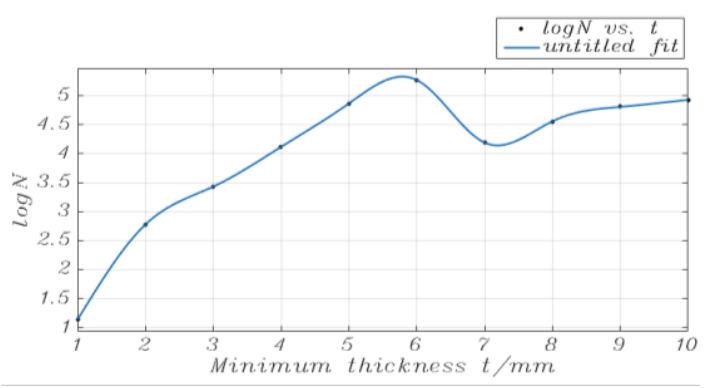

a

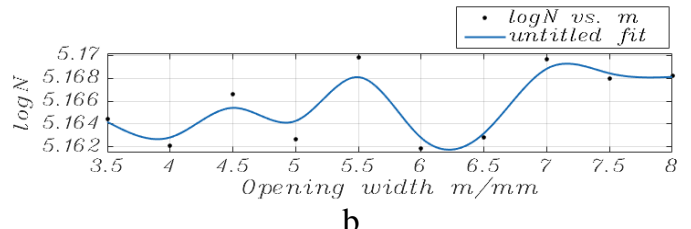

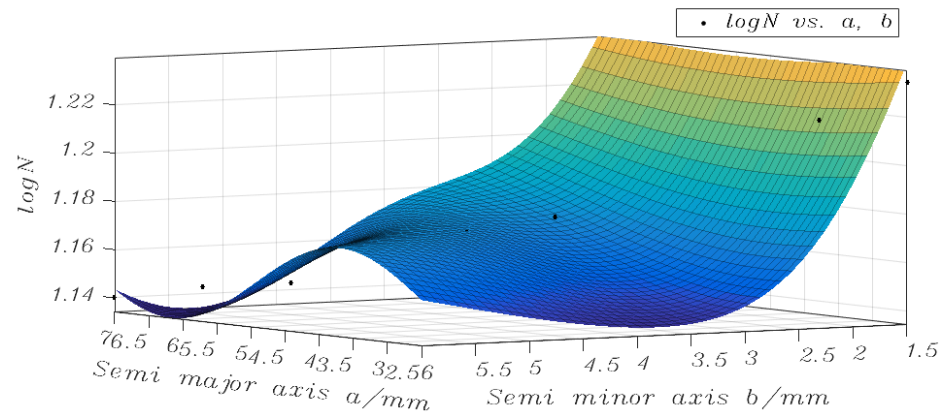

C

Fig. 9 The influence of parameters on fatigue life: a - the influence of thickness, $\mathrm{b}$ - the influence of $m, \mathrm{c}-$ the influence of $a, b$

\section{Comparison of the fatigue life and stress of U-shape flexure}

U-shape flexure hinges and circular arc flexure hinge with the same center thickness $t=6$ are selected for analysis and comparison. The notch parameters of circulararc-shaped flexure hinge are $r=12, m=24, t=6$; The model is established in ANSYS to analyze the fatigue life and stress. The analysis results of circular-arc-shaped flexure hinge are shown in Figs.10, a-b. The notch parameters and analysis results of four structures U-shape flexure hinges are shown in Table 3.

The results show that the fatigue life of the three structures of U-shape flexure hinges (U-shape with a semicircle is tangent to a line segment, U-shape with a semi-ellipse is tangent to the line segment, U-shape with an arc intersects the line segment) is higher than that of circular arc flexure hinge, while the stress of three structures of U-shape flexure hinges is less than that of circular arc flexure hinge. Therefore, the three structures of U-shape flexure hinges are more reliable than the circular arc flexure hinge.

Table 3

Fatigue life and stress of U-shape flexure hinges

\begin{tabular}{|c|c|c|c|c|c|}
\hline $\begin{array}{c}\text { Semicircle is tangent to a } \\
\text { line segment notch }\end{array}$ & $\begin{array}{c}a=b=10 \\
m=20, t=6\end{array}$ & $\begin{array}{c}a=b=9 \\
m=18, t=6\end{array}$ & $\begin{array}{c}a=b=8 \\
m=16, t=6\end{array}$ & $\begin{array}{c}a=b=7 \\
m=14, t=6\end{array}$ & $\begin{array}{c}a=b=6 \\
m=12, t=6\end{array}$ \\
\hline Fatigue life & $1.9397 \mathrm{e} 5$ & $1.9042 \mathrm{e} 5$ & $1.8603 \mathrm{e} 5$ & $1.7897 \mathrm{e} 5$ & $1.707 \mathrm{e} 5$ \\
\hline Stress & $4.8043 \mathrm{e} 6$ & $4.8543 \mathrm{e} 6$ & $4.9154 \mathrm{e} 6$ & $5.005 \mathrm{e} 6$ & $5.1129 \mathrm{e} 6$ \\
\hline \hline $\begin{array}{c}\text { Semi-ellipse is tangent to } \\
\text { the line segment notch }\end{array}$ & $\begin{array}{c}a=11.5, b=10 \\
m=20, t=6\end{array}$ & $\begin{array}{c}a=11, b=10 \\
m=18, t=6\end{array}$ & $\begin{array}{c}a=11, b=10 \\
m=20, t=6\end{array}$ & $\begin{array}{c}a=10, b=8 \\
m=16, t=6\end{array}$ & $\begin{array}{c}a=9, b=8 \\
m=16, t=6\end{array}$ \\
\hline Fatigue life & $1.8899 \mathrm{e} 5$ & $1.8297 \mathrm{e} 5$ & $1.9083 \mathrm{e} 5$ & $1.7569 \mathrm{e} 5$ & $1.7987 \mathrm{e} 5$ \\
\hline Stress & $4.87 \mathrm{e} 6$ & $4.9554 \mathrm{e} 6$ & $4.8474 \mathrm{e} 6$ & $5.0468 \mathrm{e} 6$ & $4.9912 \mathrm{e} 6$ \\
\hline \hline $\begin{array}{c}\text { Arc intersects the line } \\
\text { segment notch }\end{array}$ & $\mathrm{a}=\mathrm{b}=9$ & $a=b=8$ & $a=b=7$ & $a=b=6.5$ & $a=b=6$ \\
$m=17, t=6$ & $m=14, t=6$ & $m=10, t=6$ & $m=10, t=6$ & $m=10, t=6$ \\
\hline Fatigue life & $1.9042 \mathrm{e} 5$ & $1.8606 \mathrm{e} 5$ & $1.7898 \mathrm{e} 5$ & $1.7638 \mathrm{e} 5$ & $1.7069 \mathrm{e} 5$ \\
\hline Stress & $4.8543 \mathrm{e} 6$ & $4.9152 \mathrm{e} 6$ & $5.0048 \mathrm{e} 6$ & $5.0413 \mathrm{e} 6$ & $5.1131 \mathrm{e} 6$ \\
\hline \hline $\begin{array}{c}\text { Elliptical arc intersects } \\
\text { the line segment notch }\end{array}$ & $a=9, b=7$ & $a=8, b=7$ & $a=8, b=6$ & $a=7, b=6$ & $a=6, b=5$ \\
& $m=13.5, t=6$ & $m=13, t=6$ & $m=11, t=6$ & $m=11, t=6$ & $m=9, t=6$ \\
\hline Fatigue life & $1.655 \mathrm{e} 5$ & $1.7192 \mathrm{e} 5$ & $1.5298 \mathrm{e} 5$ & $1.6186 \mathrm{e} 5$ & $1.4747 \mathrm{e} 5$ \\
\hline Stress & $5.1749 \mathrm{e} 6$ & $5.0946 \mathrm{e} 6$ & $5.3338 \mathrm{e} 6$ & $5.2222 \mathrm{e} 6$ & $5.4046 \mathrm{e} 6$ \\
\hline
\end{tabular}

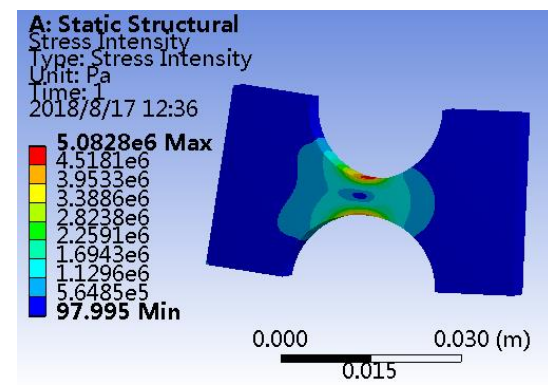

a
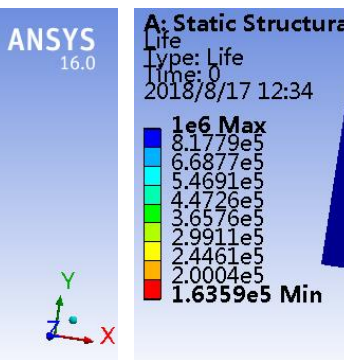

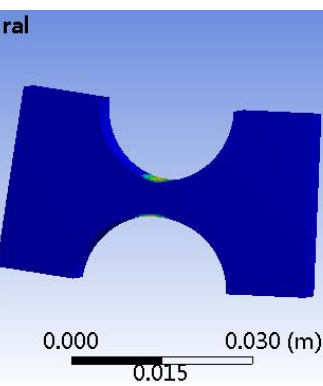

b

Fig. 10 Analysis results of circular-arc-shaped flexure hinge: $a-$ the stress distribution, $b$ - the fatigue life cloud figure 


\section{Conclusions}

The compliance equations for the U-shape flexure hinges have been derived using the Castigliano's second theorem. The influence of structure parameters on the compliance of U-shape flexure hinges is analyzed on the basis of establishing the model. The results indicate that the compliance of U-shape flexure hinge have different trends with notch parameters change, and have different sensitivity to the change of the notch parameters within the given range. The influence of notch parameters $b$ and ton compliance is more obvious than that of notch parameters $a$ and $m$.

The fatigue life is analyzed by changing the Ushape flexure hinge notch parameter. The results show that the fatigue life of flexure hinge increases gradually with the increasing of flexure hinge center thickness $t$. The fatigue life of flexure hinge increases with the increasing of hinge notch width $m$. With the increasing of the major axis of the ellipse $a$ and semi minor axis of the ellipse $b$, the fatigue life of flexure hinge fluctuates locally, the general trend is a gradual decrease. There are three structures of the U-shape flexure hinges that are more reliable than the circular arc flexure hinge, U-shape with a semi-circle is tangent to a line segment, U-shape with a semi-ellipse is tangent to the line segment, and U-shape with an arc intersects the line segment.

In summary, compliance and fatigue life of the Ushape flexure hinges is related to material properties and structure parameters, among which the influence of the notch parameters on the compliance and fatigue life is more obvious.

\section{Acknowledgement}

This research was funded by the key research and development project of Shanxi province (Grant Nos. 201803D421027, 201803D421028), the foundation of Shanxi key laboratory of advanced manufacturing technology (Grant No. XJZZ201702), and the natural science foundation of Shanxi province (Grant No. 015011060).

\section{References}

1. Yu, J. J.; Pei, X.; Bi, S. S.; Zong, G. H.; Zhang, X. M. 2010. State-of-arts of design method for flexure mechanisms, Journal of Mechanical Engineering 46(13): 2-13 (in Chinese).

2. Yu, J. J.; Hao, G. B.; Chen, G. M.; Bi, S. S. 2015. State-of-art of compliant mechanisms and their applications, Journal of Mechanical Engineering 51(13): 53-68 (in Chinese).

3. Wu, J. W.; Zhang, Y.; Cai, S.; Cui, J. W. 2018. Modeling and analysis of conical-shaped notch flexure hinges based on NURBS, Mechanism and Machine Theory 128: 560-568.

4. Yang, M.; Du, Z.J.; Dong, W. 2016. Modeling and analysis of planar symmetric superelastic flexure hinges, Precision Engineering 46: 177-183.

5. Chen, G. M., Howell, L. L. 2009. Two general solutions of torsional compliance for variable rectangular cross-section hinges in compliant mechanisms, Precision Engineering 33(3): 268-274.

6. Tian, Y.; Shirinzadeh, B.; Zhang, D.; Zhong, Y. 2010. Three flexure hinges for compliant mechanism designs based on dimension less graph analysis, Precision Engineering 34(1): 92-100.

7. Friedrich, R., Lammering, R., Rösner, M. 2014. On the modeling of flexure hinge mechanisms with finite beam elements of variable cross section, Precision Engineering 38(4): 915-920.

8. Dirksen, F.; Anselmann, M.; Zohdi, T. I.; Lammering, R. 2013. Incorporation of flexural hinge fatigue-life cycle criteria into the topological design of compliant small-scale devices, Precision Engineering 37(3): 531-541.

9. Wang, X. J.; Liu, C. L.; Gu, J. J.; Zhang, W. J. 2015. A parametric model for rotational compliance of a cracked right circular flexure hinge, International Journal of Mechanical Sciences 94-95: 168-173.

10. Li, L. J.; Zhang, D.; Guo, S.; Qu, H. B. 2017. A generic compliance modeling method for two-axis elliptical-arc-filleted flexure hinges, Sensors 17(9): 21-54.

11. Wu, J. W.; Cai, S; Cui, J. W.; Tan, J. B. 2015. A generalized analytical compliance model for cartwheel flexure hinges, Review of Scientific Instruments 86: 105003(1-11).

12. Tian, Y.; Shirinzadeh, B.; Zhang, D. Closed-form compliance equations of filleted $\mathrm{V}$-shaped flexure hinges for compliant mechanism design, Precision Engineering 34(3): 408-418.

13. Chen, G. 2014. Generalized equations for estimating stress concentration factors of various notch flexure hinges, Journal of Mechanical Design 136(3): 252-261.

14. Xu, N.; Dai, M.; Zhou, X.Q. 2017. Analysis and design of symmetric notch flexure hinges, Advances in Mechanical Engineering 9(11): 1-12.

15. Li, L. J.; Zhang, D.; Guo, S.; Qu, H. B. 2019. Design, modeling, and analysis of hybrid flexure hinges, Mechanism and Machine Theory 131: 300-316.

16. Wang, X. J.; Liu, C. L.; Gu, J. J.; Zhang, W. J. 2015. A parametric model for rotational compliance of a cracked right circular flexure hinge, International Journal of Mechanical Sciences 94-95: 168-173.

17. Li, Q.; Pan, C. Y.; Xu, X. J. 2013. Closed-form compliance equations for power-function-shaped flexure hinge based on unit-load method, Precision Engineering $37: 135-145$.

18. Meng, Q.; Li, Y.; Xu, J. 2013. New empirical stiffness equations for corner-filleted flexure hinges, Mechanical Sciences 4(2): 345-356.

19. Li, T. M.; Zhang, J. L.; Jiang, Y. 2015. Derivation of empirical compliance equations for circular flexure hinge considering the effect of stress concentration, International Journal of Precision Engineering and Manufacturing 16(8): 1735-1743.

20. Lobontiu, N.; Paine, J. S. N.; Garcia, E.; Goldfarb, M. 2002. Design of symmetric conic-section flexure hinges based on closed-form compliance equations, Mechanism and Machine Theory 37: 477-498. 
J.J. Liang, R.Q. Li, S.P. Bai, Q. Li, F.P. Ning, S.H. Kang

COMPLIANCE AND FATIGUE LIFE ANALYSIS OF U-SHAPE FLEXURE HINGE

\section{S u m m a r y}

This paper establishes four models of U-shape flexure hinges with different notch shapes and structure parameters, and presents the close-form compliance equations for the four structure types of U-shape flexure hinges. The compliance of the flexure hinges is developed based on the Castiglione's second theorem and calculus theory. A relationship between compliances and structure parameters is deduced using the models. The influences of the notch structure parameters on the compliance of the flexure hinges are investigated. Moreover, fatigue life of U-shape flexure hinges is studied by finite element analysis, the results show that the fatigue life of flexure hinge increases gradually with the increasing of flexure hinge center thickness $t$ and hinge notch width $m$. With the increasing of the major axis of the ellipse $a$ and semi minor axis of the ellipse $b$, the fatigue life of flexure hinge fluctuates locally, the general trend is a gradual decrease. The stress and fatigue life of U-shape flexure hinges and arc flexure hinge are compared. The results show that the reliability of U-shape flexure hinge is higher than that of circular arc flexure hinge.

Keywords: compliance, fatigue life, reliability, U-shape flexure hinge.

Received February 04, 2019

Accepted November 21, 2019 